\title{
Inflammatory pseudotumor of eyelid: a probable IgG4-related sclerosing disease clinically mimicking eyelid pilomatrixoma
}

\author{
Youn Joo Choi ${ }^{1}$, Min Joung Lee ${ }^{2}$, Namju Kim ${ }^{3,6}$, Ho-Kyung Choung ${ }^{4,6^{*}}$, Sang In Khwarg ${ }^{5,6}$ and Ji Eun Kim
}

\begin{abstract}
Background: Ocular adnexal lgG4-related sclerosing disease (IgG4-SD) has been categorized as a novel disease entity. It is characterized by stromal sclerosis and an infiltration of mass-forming lymphoplasmic cells containing many IgG4-positive plasma cells. Although ocular adnexal tissue involvement has been increasingly reported, a focal nodular sub-brow mass is not typical in an IgG4-SD presentation. We report a rare case of probable ocular adnexal IgG4-SD that clinically mimicked eyelid pilomatrixoma.
\end{abstract}

Case presentation: A 42-year-old woman presented with a nodular mass in her left sub-brow area. The initial clinical impression of her lesion was eyelid pilomatrixoma. However, the final pathologic diagnosis was IgG4-SD, but extranodal marginal zone B-cell lymphoma could not be excluded. The patient underwent testing to determine tumor malignancy and systemic lgG4-SD involvement. Laboratory testing showed normal lgG and lgG4 serum levels and imaging revealed no remarkable findings. Oral prednisolone was administered and slowly tapered to manage the possible remnant lesion and to prevent disease recurrence. Two years after initiating therapy, there was no evidence of relapse. The patient is under close surveillance for signs of recurrence, systemic involvement, and potential malignant transformation.

Conclusions: We found an unusual case of probable ocular adnexal IgG4-SD, which presented as a unilateral restricted mass involving the sub-brow area. Although the mass was surgically removed, systemic steroid treatment and long-term surveillance were initiated due to the possibility of recurrence, the potential association with systemic disease, and the potential development of extranodal mucosa-associated lymphoid tissue (MALT) lymphoma.

Keywords: Eyelid, IgG4-related sclerosing disease, Ocular adnexa

\section{Background}

Ocular adnexal IgG4-related sclerosing disease (IgG4-SD) has been categorized as a novel disease entity that may account for a certain proportion of idiopathic ocular and periocular inflammatory lesions. It is characterized by stromal sclerosis and an infiltration of mass-forming lymphoplasmic cells containing many IgG4-positive plasma cells. It can involve various organs including pancreas, bile duct, retroperitoneal soft tissues, liver, thyroid, lung and salivary glands, either singly or systematically $[1,2]$. Although the

\footnotetext{
*Correspondence: hokyung214@gmail.com

${ }^{4}$ Department of Ophthalmology, Seoul Metropolitan Government Seoul National University Boramae Medical Center, 39, Boramae-gil, Dongjak-gu, Seoul 156-707, Korea

${ }^{6}$ Department of Ophthalmology, College of Medicine, Seoul National University, Seoul, Korea

Full list of author information is available at the end of the article
}

prototype of IgG4-SD in ophthalmologic disorders is bilateral dacryoadenitis accompanied by sialadenitis (formerly termed Mikulicz disease), involvement of other ocular adenexal tissues such as orbital fat, extraocular muscles, and lacrimal sac has been increasingly reported in the literature [3-5]. However, a focal nodular sub-brow mass is not typical in an IgG4-SD presentation. We report a rare case of probable ocular adnexal IgG4-SD that clinically mimicked eyelid pilomatrixoma.

\section{Case presentation}

A 42-year-old woman with no significant past medical history presented with a painless nodular mass in her left upper lid, which had had slowly enlarged over 1 year. On clinical examination, a non-tender, nodular mass $(5.5 \mathrm{~mm} \times 4.5 \mathrm{~mm})$ was palpable in the left sub-brow region and was freely mobile over subcutaneous tissue (Figure 1A). A diagnosis of 

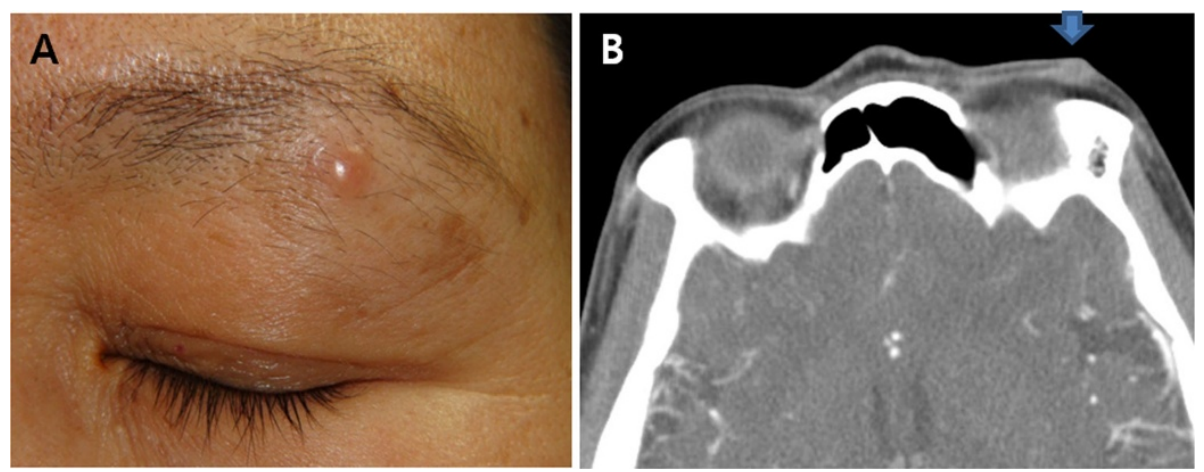

Figure 1 Clinical presentation. (A) Clinical photograph of a patient presenting with a $5.5 \mathrm{~mm} \times 4.5 \mathrm{~mm}$ soft non-tender nodular mass with no overlying skin change. (B) An orbital CT scan showing a 7-mm nodular homogenous enhancing mass adherent to the skin of the left upper lid (arrow).

presumed pilomatrixoma of the eyelid was made. Computed tomography with contrast of the orbit showed a 7-mm nodular homogenous enhancing mass that was adherent to the left upper lid skin (Figure 1B). This implied that the tumor had originated from either skin or a dermal appendage.

A total excisional biopsy was scheduled. Unfortunately, the tumor's infiltrative growth pattern made it difficult to completely excise it from surrounding tissues. The tumor was pinkish to brown in color, poorly circumscribed, and had a rubbery surface.

Histology revealed that the mass had a dense lymphocytic infiltration with lymphoid follicles, moderate plasma cell infiltration with a few eosinophils, and interstitial fibrosis (Figure 2A, B). Immunohistology showed increased CD20+ B-cells with slight marginal zone expansion, focal $\mathrm{CD} 3+\mathrm{T}$ cell infiltration, a polyclonal plasma cell population with an even distribution of kappa and lambda light chains, and a low Ki-67 proliferative index (5\% of all lymphoid cells). The number of IgG4+ plasma cells, counted in a high power field (HPF: X10 eyepiece and x 40 objective lenses), was $>50$ and the IgG4/IgG ratio was $50 \%$
(Figure 2C). The resulting final pathologic diagnosis was inflammatory pseudotumor with increased IgG4+ plasma cells; possible association with IgG4-SD, but extranodal marginal zone B-cell lymphoma could not be excluded.

Therefore, the patient underwent testing to determine tumor malignancy and systemic IgG4-SD involvement. Laboratory testing showed normal IgG and IgG4 serum levels. [1030 mg/dL (reference range, 700-1600), $21.1 \mathrm{mg} / \mathrm{dL}$ (reference range, 6.1-121.4), respectively]. Serum Lactate dehydrogenase concentration was within normal range. Except for a $3 \mathrm{~cm}$ sized uterine myoma, imaging studies including chest $\mathrm{CT}$, abdomen and pelvic $\mathrm{CT}$, and positron emission tomography scanning demonstrated no remarkable findings.

For the management of possible remnant lesion and prevention of recurrence of disease, oral prednisolone was administered at a $40 \mathrm{mg} /$ day initial dose and was slowly tapered to a $2.5 \mathrm{mg} /$ day maintenance dose. One year after initiating therapy, there was no evidence of relapse. The patient is under close surveillance for signs of recurrence, systemic involvement, and potential malignant transformation.
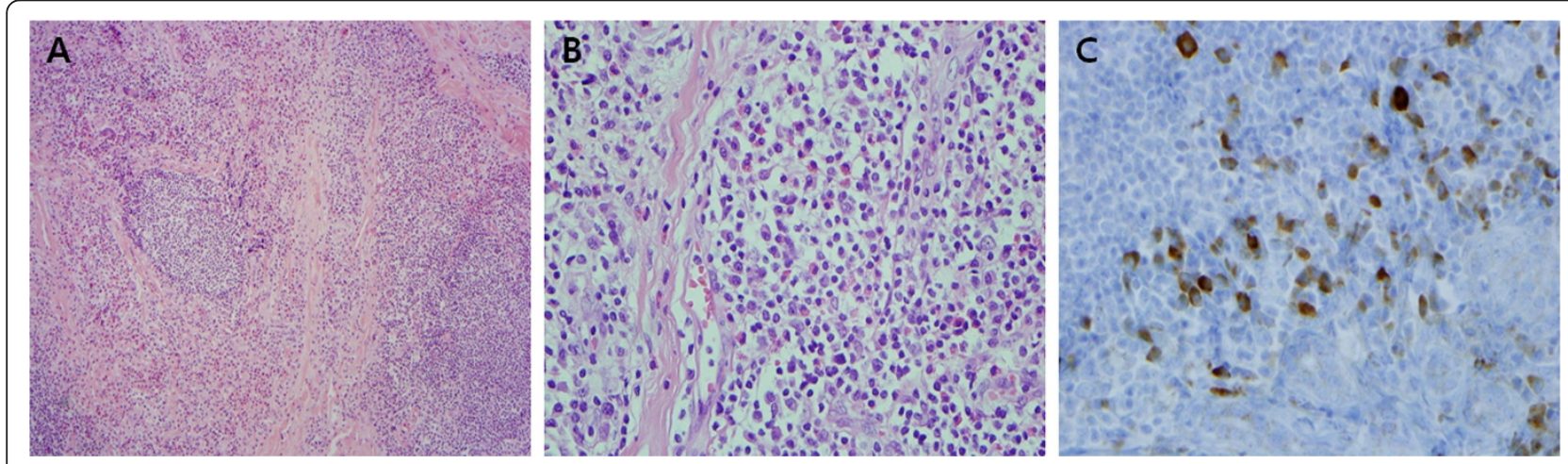

Figure 2 Histopathology. Biopsied specimen shows lymphoid nodules and interstitial fibrosis (A) hematoxylin-eosin staining, $\times 100$ magnification). Under higher magnification, interfollicular spaces are filled with plasma cells and eosinophils (B) hematoxylin-eosin, magnification $\times 400$ ). (C) Infiltration of IgG4-positive plasma cells numbered >50 in a high-power field (anti-lgG4 antibody, magnification $\times 400$ ). 


\section{Discussion}

Recognition of IgG4-SD is clinically important because this disease is, theoretically, a systemic disorder. Steroid therapy has been proposed to be effective, but relapse occurs frequently. Furthermore, recent studies suggest that IgG4+ plasma cells might be involved in the pathogenesis of mucosa-associated lymphoid tissue (MALT) lymphoma [6-8]. Moreover, many ocular adnexal MALT lymphoma cases are wrongly categorized as IgG4-SD, illustrating the difficulty of distinguishing between MALT lymphoma and IgG4-SD [7,9,10]. In our case, the lesion's clinical appearance was typical of an eyelid pilomatixoma, a benign tumor originating from the hair follicle matrix that occurs frequently in the sub-brow area in the pediatric age group. Clinically, it appears as a slowly enlarging, non tender nodular mass which is freely movable over the subcutaneous tissue. The present case demonstrates the importance of pathologic confirmation, even when clinical examination suggests that a lesion is likely benign with clinically typical presentation.

Two characteristics IgG4-SD include dense sclerosis with abundant IgG4+ plasma cell infiltration and serum IgG4 level elevation [1]. In this case, as the clinical finding of unilateral focal subbrow mass was not typical presentation of IgG4-SD, the autoantibody profile including serum IgG4 level was not evaluated at initial presentation. After the pathologic confirmation of IgG4$\mathrm{SD}$, that is, two weeks after excisional biopsy, the postoperative serum IgG4 levels were checked. The timing of the measurement would be one of the possible explanations of the normal serum IgG4 level of this patient, as grossly no residual lesion existed postoperatively. Even though there was no preoperative evidence of serum IgG4 elevation in our patient, this case was given a probable diagnosis of IgG4-SD of the ocular adnexa based on the comprehensive diagnostic criteria for IgG4-SD (See the 'Comprehensive diagnostic criteria for IgG4-related sclerosing disease' section). It is true that serum IgG4 levels are frequently elevated in patients with IgG4-SD and this finding can be a useful diagnostic tool, however, not all of the reported cases with IgG4-SD revealed elevated serum IgG4 concentration [11-14]. Yamamoto et al. showed that high serum IgG4 levels are not specific to IgG4-SD [14].

\section{Comprehensive diagnostic criteria for IgG4-related sclerosing disease, 2011}

1. Clinical examination showing characteristic diffuse/ localized swelling or masses in single or multiple organs

2. Hematological examination shows elevated serum IgG4 concentrations( $135 \mathrm{mg} / \mathrm{dl}$ )
3. Histopathologic examination shows:

(1) Marked lymphocyte and plasmacyte infiltration and fibrosis.

(2) Infiltration of IgG4+ plasma cells: ratio of IgG4+/IgG+ cells $>40 \%$ and $>10$ IgG $4+$ plasma cells/HPF.

Definite: 1$)+2)+3)$

Probable: 1$)+3$ )

Possible: 1$)+2$ )

\section{Conclusions}

We found an unusual case of probable ocular adnexal IgG4-SD, which presented as a unilateral restricted mass involving the sub-brow area. Although the mass was surgically removed, systemic steroid treatment and longterm surveillance was initiated due to the possibility of recurrence, the potential association with systemic disease, and the potential development of extranodal MALT lymphoma.

\section{Consent}

Written informed consent was obtained from the patient for publication of this Case Report and any accompanying images. A copy of the written consent is available for review by the Editor of this journal.

\section{Abbreviations \\ IgG4-SD: IgG4-related sclerosing disease; MALT: Mucosa-associated lymphoid tissue; HPF: High power field.}

\section{Competing interests}

The authors declare that they have no competing interests.

\section{Authors' contributions}

HK conceived of the study, performed the excisional biopsy, and has given final approval of the version to be published. JE is a pathologist who carried out the histopatholic diagnosis and helped to draft the manuscript. YJ drafted the manuscript. MJ and NJ were involved in drafting the manuscript. SI critically revised the manuscript. All authors read and approved the final manuscript.

\section{Acknowledgements}

None of the authors have received funding for this study.

\section{Author details}

'Department of Ophthalmology, Kangdong Sacred Heart Hospital, Hallym University Medical Center, Seoul, Korea. ${ }^{2}$ Department of Ophthalmology, Hallym Sacred Heart Hospital, Anyang, Korea. ${ }^{3}$ Department of Ophthalmology, Seoul National University Bundang Hospital, Seongnam, Korea. ${ }^{4}$ Department of Ophthalmology, Seoul Metropolitan Government Seoul National University Boramae Medical Center, 39, Boramae-gil, Dongjak-gu, Seoul 156-707, Korea. ${ }^{5}$ Department of Ophthalmology, Seoul National University Hospital, Seoul, Korea. ${ }^{6}$ Department of Ophthalmology, College of Medicine, Seoul National University, Seoul, Korea. ${ }^{7}$ Department of Pathology, Seoul Metropolitan Government Seoul National University Boramae Medical Center, Seoul, Korea.

Received: 4 July 2014 Accepted: 6 February 2015

Published online: 08 March 2015 


\section{References}

1. Umehara H, Okazaki K, Masaki Y, Kawano M, Yamamoto M, Saeki T, et al. Comprehensive diagnostic criteria for lgG4-related disease (lgG4-RD), 2011. Mod Rheumatol. 2012;22:21-30.

2. Stone JH, Zen Y, Deshpande V. IgG4-related disease. N Engl J Med. 2012;366:539-51

3. Wallace ZS, Khosroshahi A, Jakobiec FA, Deshpande V, Hatton MP, Ritter J, et al. IgG4-related systemic disease as a cause of "idiopathic" orbital inflammation, including orbital myositis, and trigeminal nerve involvement. Surv Ophthalmol. 2012;57:26-33.

4. Pasquali T, Schoenfield L, Spalding SJ, Singh AD. Orbital inflammation in IgG4-related sclerosing disease. Orbit. 2011;30:258-60.

5. da Fonseca FL, Ramos Rde I, de Lima PP, Nogueira AB, Matayoshi S. Unilateral eyelid mass as an unusual presentation of ocular adnexal lgG4-related inflammation. Cornea. 2013;32:517-9.

6. Sato Y, Takata K, Ichimura K, Tanaka T, Morito T, Tamura M, et al. IgG4-producing marginal zone B-cell lymphoma. Int J Hematol. 2008:88:428-33.

7. Kubota T, Moritani S, Yoshino T, Nagai H, Terasaki H. Ocular adnexal marginal zone B cell lymphoma infiltrated by lgG4-positive plasma cells. J Clin Pathol. 2010;63:1059-65.

8. Cheuk W, Yuen HK, Chan AC, Shih LY, Kuo T, Ma MW, et al. Ocular adnexal lymphoma associated with lgG4+ chronic sclerosing dacryoadenitis: a previously undescribed complication of IgG4-related sclerosing disease. Am J Surg Pathol. 2008;32:1159-67.

9. Karamchandani JR, Younes SF, Warnke RA, Natkunam Y. IgG4-related systemic sclerosing disease of the ocular adnexa: a potential mimic of ocular lymphoma. Am J Clin Pathol. 2012;137:699-711.

10. Go H, Kim JE, Kim YA, Chung HK, Khwarg SI, Kim CW, et al. Ocular adnexal IgG4-related disease: comparative analysis with mucosa-associated lymphoid tissue lymphoma and other chronic inflammatory conditions. Histopathology. 2012;60:296-312.

11. Mizutani S, Suzuki H, Yoshida H, Arima Y, Kitayama $Y$, Uchida E. A case of IgG4-related sclerosing cholangitis with a normal serum lgG4 level: report of a case. J Nippon Med Sch. 2012;79:367-72.

12. Ohno K, Sato Y, Ohshima Kl, Takata K, Ando M, Abd Al-Kader L et al.. IgG4-related disease involving the sclera. Mod Rheumatol. 2012;24:195-8.

13. Masri KR, Shaver T. IgG4-related systemic disease: an uncommon presentation for an uncommon disease. Rheumatol Int. 2013;33:1865-6.

14. Yamamoto M, Tabeya T, Naishiro $Y$, Yajima H, Ishigami $K$, Shimizu $Y$, et al Value of serum lgG4 in the diagnosis of lgG4-related disease and in differentiation from rheumatic diseases and other diseases. Mod Rheumatol. 2012;22:419-25.

\section{Submit your next manuscript to BioMed Central and take full advantage of:}

- Convenient online submission

- Thorough peer review

- No space constraints or color figure charges

- Immediate publication on acceptance

- Inclusion in PubMed, CAS, Scopus and Google Scholar

- Research which is freely available for redistribution 